\title{
USO DE FUNCIONES DE GUMBEL PARA EL AJUSTE Y DECONVOLUCIÓN DE TERMOGRAMAS DE ANÁLISIS TÉRMICO DIFERENCIAL
}

\section{USE OF GUMBEL DISTRIBUTION FUNCTIONS FOR THE FITTING AND DECONVOLUTION OF DIFFERENTIAL THERMAL ANALYSIS THERMOGRAMS}

Jesús González-Laprea ${ }^{1 *}$, Carlos Durante R. ${ }^{2}$ \& José Darias G. ${ }^{3}$

Recibido: 6 de febrero 2020 / Aceptado: 12 de junio 2020

Publicado en línea: 19 de junio 2020

DOI: $10.26807 / i a . v 8 i 2.131$

Palabras claves: Ajuste de datos experimentales, Análisis Térmico Diferencial (ATD), deconvolución de señales, funciones de Gumbel, transiciones de fase.

Keywords: Experimental data fitting, Differential Thermal Analysis (DTA), Gumbel functions, phase transitions, signal deconvolution.

1 Facultad de Ciencias Exactas y Naturales, Escuela de Ciencias Físicas y Matemáticas, Pontificia Universidad Católica del Ecuador. Quito, Ecuador. (*correspondencia: jegonzalezl@puce.edu.ec)

2 Laboratorio de Ciencia de Materiales, Departamento de Física, Facultad Experimental de Ciencias, Universidad del Zulia. Maracaibo, Venezuela. (durincarlos@gmail.com)

3 Laboratorio de Óptica y Fluidos, Universidad Simón Bolívar, Caracas, Venezuela. (jrdarias@usb.ve) 


\section{RESUMEN}

Se presenta una propuesta para el ajuste empírico de curvas correspondientes a transiciones de fase en mediciones obtenidas por Análisis Térmico Diferencial (ATD). La familia de funciones que se plantean para el ajuste se conoce como funciones Gumbel; estas funciones tienen características de asimetría que se asemejan a las de los picos típicos de ATD, a diferencia de funciones simétricas usualmente utilizadas para el ajuste de picos, como Gaussianas o Lorentzianas. El proceso de ajuste se llevó a cabo con mediciones de ATD realizadas en muestras de compuestos $\ln _{\mathrm{x}} \mathrm{Mn}_{(1-\mathrm{x})} \mathrm{Sb}$, los cuales exhiben la presencia de dos fases; InSb y MnSb, en las que dependiendo del valor de x se observan transiciones de fase que pueden ser o no simultáneas en temperatura. Al trabajar con curvas de transiciones de fase individuales, las funciones de Gumbel ajustan las curvas con coeficientes de correlación lineal $\left(\mathrm{R}^{2}\right)$ superiores a 0,98, tanto para transiciones de fusión como para transiciones de solidificación, lo que evidencia el correcto ajuste de los datos experimentales. Se demuestra la valía de este tipo de ajustes al ser empleados en un sistema donde existen transiciones de fase simultáneas en temperatura, en este caso se muestra cómo, con las funciones de Gumbel, es posible separar estas señales superpuestas. Una vez separadas se podría tener acceso a información como la temperatura de transición o a la entalpía de transformación de cada uno de los compuestos presentes en la mezcla, algo que actualmente es muy complicado de hacer para señales simultáneas de ATD.

\section{ABSTRACT}

We present a proposal for the empirical fit of curves corresponding to phase transitions in measurements obtained using Differential Thermal Analysis (DTA). The family of functions for fit is known as the Gumbel functions. These functions have asymmetrical characteristics that resemble those of typical DTA peaks, different from the symmetrical functions usually used for the adjustment of peaks (such as Gaussian or Lorentzian). The adjustment process was performed with DTA measurements carried out with samples of $\ln _{x} M n_{(1-x)}$ Sb compounds, which 
show the presence of two phases, InSb and MnSb. In these phases, depending on the value of $x$, phase transitions are observed that may or may not be simultaneous in temperature. When working with individual phase transition curves, Gumbel functions fits the curves with linear correlation coefficients $\left(R^{2}\right)$ higher than 0.98 -for both fusion and solidification transitions- which demonstrates the experimental data's proper fit. This type of fit's value is demonstrated by its use in a system where there are simultaneous phase transitions in temperature. In this case it is shown how, with the Gumbel functions, it is possible to separate these superimposed signals. These independent signals may provide access to information such as the phase transition temperature and the enthalpy of transformation for each of the compounds present in the mixture, something presently difficult to attain in simultaneous DTA signals.

\section{INTRODUCCIÓN}

El Análisis Térmico Diferencial (ATD) es una técnica de amplio uso para la caracterización de materiales sólidos, se basa en la comparación entre las temperaturas de la muestra en estudio y un material referencial inerte con una capacidad calórica similar, mientras ambas experimentan un proceso de intercambio de calor (Akash \& Rehman, 2020; Školáková, Prǔša, \& Novák, 2020). Cuando la muestra experimenta alguna reacción o transformación, y la tasa a la que se modifica su temperatura es alterada, la diferencia entre las dos temperaturas es evidenciada como una señal en el termograma obtenido. Así, si se produce un proceso endotérmico durante el calentamiento de la muestra (una fusión, por ejemplo), ésta reducirá su velocidad de calentamiento mientras ocurre dicho proceso, generando un pico de ATD. Un cambio en la capacidad calórica o la conductividad térmica del material producirá cambios en la pendiente de la señal (Akash \& Rehman, 2020; Charsley et al., 2019; Kramer, 1992).

Este último elemento es definitorio para las señales de ATD; si la muestra, a lo largo del proceso de calentamiento o enfriamiento, experimenta cambios de fase, la capacidad caló- 
rica de ésta, muy probablemente será diferente antes y después del proceso, por lo que normalmente los picos asociados tienden a ser marcadamente asimétricos, presentando pendientes diferentes en cada uno de sus flancos (Akash \& Rehman, 2020; Janssen, Nicasio, Dudley, Xia, \& Simonson, 2020; Kramer, 1992).

La función principal de un análisis por ATD es identificar procesos térmicos; en procesos endotérmicos (fusión, vaporización, sublimación, desorción o cambios de fase cristalina), la temperatura de la muestra será inferior a la de la referencia, por lo que se observarán picos hacia abajo en la señal, mientras que para procesos exotérmicos (absorción, nucleación, cristalización, o cambios en fases metaestables), se observarán picos hacia arriba (Akash \& Rehman, 2020; Charsley et al., 2019; Kramer, 1992; Verma, Devi, Sharma, \& Jensen, 2011). En cualquiera de los casos, el área bajo la curva temperatura - tiempo para cada una de estas señales estará dada por la entalpía de transformación correspondiente al proceso suscitado (Akash \& Rehman, 2020; Borchardt \& Daniels, 1957; Charsley et al., 2019).
Cuando la muestra en estudio consiste en la mezcla de dos o más fases, los termogramas de ATD suelen exhibir un número igual o menor de señales, correspondientes a las transformaciones (químicas o físicas) ocurridas en cada una de ellas. Dado que muchas veces estas señales ocurren en intervalos de temperatura similares, se recurre a reducir considerablemente la velocidad de calentamiento y así lograr que las transiciones no se superpongan entre sí.

Esta metodología trae consigo dos limitantes; en primer lugar, está demostrado que al incrementar la velocidad de calentamiento el desfase entre la temperatura de la referencia y la temperatura de la muestra se incrementa, mejorando la señal en el ATD, por lo que al reducir la rapidez del calentamiento se puede perder información. Esto crea un compromiso al momento de escoger la tasa de calentamiento adecuada para el estudio (Akash \& Rehman, 2020; Kramer, 1992).

La segunda limitante está en la naturaleza intrínseca del compuesto, en donde las fases pueden estar íntimamente ligadas (como por ejemplo en mezclas eutécticas de sistemas bina- 
rios con miscibilidad total en las fases líquida y sólida) donde es imposible obtener señales independientes para las transformaciones ocurridas (Janssen et al., 2020; Kramer, 1992).

\section{La función de distribución de Gum- bel}

La función de distribución de Gumbel (Gumbel, 1954) es una distribución de densidad de probabilidad, comúnmente utilizada para el estudio de la distribución de valores extremos dentro de un conjunto de datos (Chang, 2015; Hershfield, 1973), se utiliza, por ejemplo, para el estudio de picos de pluviosidad o niveles máximos de ríos, tamaños extremos en una población o valores mínimos o máximos en análisis financieros, entre mucha otras aplicaciones (Chang, 2015; Gorgoso-Varela \& Rojo-Alboreca, 2014; Gumbel, 1954; Hershfield, 1973; Nadarajah, 2006). Dado que es una función que estudia valores extremos, se caracteriza por su alta asimetría (Chang,
2015), como sería de esperar para valores extremos, se obtienen distribuciones de colas largas hacia la izquierda y caídas abruptas a la derecha para distribuciones de valores mínimos (Figura 1) y, el caso opuesto (colas largas a la derecha y una caída rápida a cero para la derecha) para las distribuciones de valores máximos (Chang, 2015; Gorgoso-Varela \& Rojo-Alboreca, 2014; Gumbel, 1954).

La función de densidad de probabilidad de Gumbel está dada por la ecuación (1):

$$
f(x)=y_{0}+\frac{1}{\beta} \exp \left\{\exp \left[-\left(\frac{x-\alpha}{\beta}\right)\right]+\left(\frac{x-\alpha}{\beta}\right)\right\}
$$

donde $\alpha$ y $\beta$ son respectivamente los parámetros de localización y escala de la función, $y_{0}$ es un parámetro de ajuste vertical. En la Figura 1, se puede observar cómo se modifica la forma de la curva ajustando los valores de estos parámetros. 


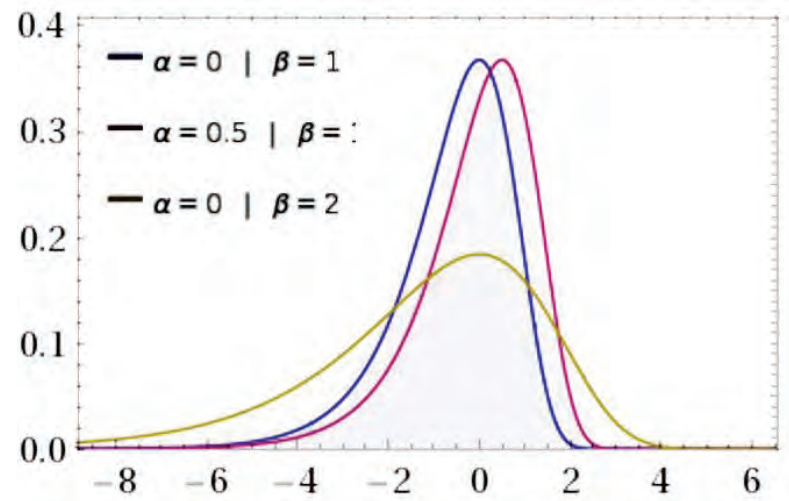

Figura 1. Función de Gumbel para distribución de mínimos (generada en Wolfram Alpha), tomando tres valores diferentes de los parámetros $\alpha$ y $\beta$.

Se puede observar la asimetría de la función en su cola larga hacia la izquierda y cómo se puede ajustar su centro ajustando el parámetro $\alpha$

El objetivo del presente trabajo es utilizar las características de asimetría de las funciones de Gumbel, para realizar ajustes empíricos de los picos correspondientes a señales de análisis térmico diferencial (ATD). De esta forma se pueden deconvolucionar picos de mezclas de sustancias que presenten transiciones de fase en intervalos similares de temperaturas, y así se podría suministrar información de lo que ocurre individualmente en cada uno de los constituyentes de la muestra en estudio.

\section{MATERIALES Y MÉTODOS}

Para implementar las funciones Gumbel en el ajuste de picos de ATD, se realizaron medidas sobre una muestra de InSb con incrustaciones de $\mathrm{MnSb}$ en dos proporciones diferentes: $\ln _{0,80} \mathrm{Mn}_{0,20} \mathrm{Sb}$ y $\operatorname{In}_{0,90} \mathrm{Mn}_{0,10} \mathrm{Sb}$.
La inserción de Mn en semiconductores de la familia III-V (como el InSb) se ha ido desarrollando en los últimos años, con la intención de fabricar semiconductores con propiedades magnéticas para aplicaciones en es- 
pintrónica, dirigidos principalmente a su uso en computación cuántica, para la cual se están desarrollando semiconductores magnéticos diluidos (DMS por sus siglas en inglés) de alta temperatura de Curie (TC) (Awschalom, Loss, \& Samarth, 2013; Katayama-Yoshida \& Sato, 2003). Justamente es la alta TC de estos materiales $(\mathrm{TC}=173 \mathrm{~K})$, lo que los hace especialmente útiles para aplicaciones como comunicación satelital o circuitos integrados (Liu, Li, \& Zeng, 2010).

Las muestras fueron preparadas a partir de una mezcla de elementos respetando las proporciones estequiométricas del compuesto deseado y utilizando las cantidades para obtener un lingote de $4 \mathrm{~g}$. Se utilizó indio (In) en trozos con un grado de pureza de 99,999\%, antimonio (Sb) en granos con un grado de pureza de 99,9999\% y manganeso (Mn) con un grado de pureza de 99,99\%. Los materiales fueron colocados en una cápsula de cuarzo con calidad para semiconductores, tratada adecuadamente para evitar la presencia de impurezas. Las paredes internas de la cápsula fueron cubiertas con grafito para evitar la presencia de fracturas en el lingote por la adhesión de éste al cuarzo. Los elementos se agregaron en la capsula grafitada para colocarla en un sistema de alto vacío $\left(\sim 10^{-6}\right.$ torr) por 24 horas antes de ser sellada. Para la elaboración del programa de síntesis se tomó en cuenta los puntos de fusión de los elementos constituyentes y las transiciones de fase en el proceso de enfriamiento controlado. Se calentó desde la temperatura ambiente $\left(\sim 25{ }^{\circ} \mathrm{C}\right)$ hasta $1150{ }^{\circ} \mathrm{C}$ a $10{ }^{\circ} \mathrm{C} / \mathrm{h}$, manteniéndose esta temperatura por 36 horas, agitando la capsula varias veces mientras transcurría este periodo de tiempo, para que los elementos ya fundidos (In y Sb) se mezclen adecuadamente y se forme antimoniuro de indio en fase liquida. A pesar de que la temperatura a la cual es Ilevada la muestra inicialmente no alcanza el punto de fusión del manganeso $\left(\sim 1260^{\circ} \mathrm{C}\right)$ éste es incorporado a la muestra mediante difusión, terminando así la etapa de calentamiento. Se enfrió el horno hasta $500{ }^{\circ} \mathrm{C}$ a $5{ }^{\circ} \mathrm{C} / \mathrm{h}$ pasando así por las posibles transiciones de fase que pudiera presentar el compuesto ya formado. Una vez que el horno llega a $500{ }^{\circ} \mathrm{C}$, se realizó un recocido térmico (annealing) durante 96 horas. 
Para verificar la presencia de ambas fases se realizó un estudio de difracción de rayos $X$ sobre las muestras; se realizó difracción de rayos $X$ en polvo a temperatura ambiente, Ilevada a cabo en un difractómetro PANalytical X'Pert PRO con ánodo de cobre $(\lambda=1,54060 \AA)$ y geometría Bragg-Brentano. La medida se realizó en el intervalo de $2 \theta$ entre $5^{\circ}$ y $100^{\circ}$ con un paso de $0,0170^{\circ}$, mientras que el tiempo de toma de medidas fue de 1,9050 s/paso.

Para el análisis térmico diferencial se empleó un equipo marca Shimadzu, modelo DTA-50, dotado con termopares tipo Pt-Pt/10 \%Rh, controlado por un computador por medio del Software TA-60WS. La muestra triturada es introducida en una cápsula de cuarzo sellada al vacío ( 10-6 torr), con el fin de minimizar la oxidación y la pérdida de material al momento de monitorear la muestra. Se tomaron las previsiones para obtener el mejor contacto térmico posible entre el termopar y el portamuestra. Como referencia inerte se utilizó polvo de alúmina $\left(\mathrm{Al}_{2} \mathrm{O}_{3}\right)$ de alta pureza, encapsulado en condiciones idénticas a las anteriores.

\section{RESULTADOS}

\section{Análisis de rayos $\mathrm{X}$}

En el análisis del patrón de difracción de rayos $X$ en polvo (Figura 2), se utilizó el programa WinPLOTR (Roisnel \& Rodríquez-Carvajal, 2001) para leer las posiciones $2 \theta$ de los picos y obtener los índices de Miller, la es- tructura cristalina y las figuras de mérito.

La indexación mostró una fase mayoritaria con estructura cúbica tipo zincblenda ( $\mathrm{ZnS})$. Otras fases fueron encontradas (Figura 2, $2 \theta=29$ y 45 grados) correspondiendo a MnSb. 


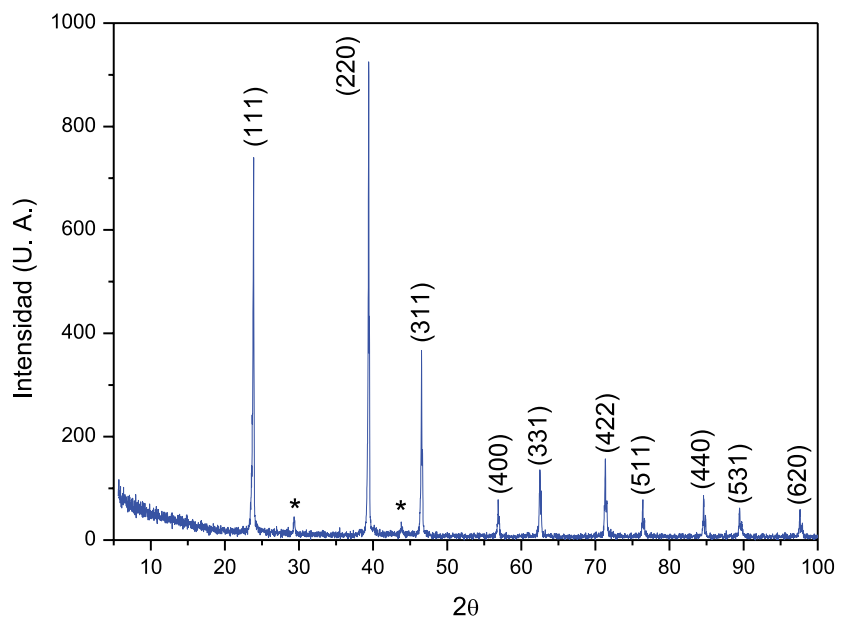

Figura 2. Difractograma mostrando los índices de Miller (hkl) de las reflexiones del compuesto InSb dopado con $\mathrm{Mn}$ en el lingote de InSb/MnSb. Los picos marcados con * corresponden a MnSb rico en Sb

\section{Mediciones de ATD}

Las medidas de ATD se realizaron en corridas de $15{ }^{\circ} \mathrm{C} / \mathrm{min}$ para el $\mathrm{In}_{0,80} \mathrm{Mn}_{0,20} \mathrm{Sb}$ y $10{ }^{\circ} \mathrm{C} / \mathrm{min}$ para el $\mathrm{In}_{0,90} \mathrm{Mn}_{0,10} \mathrm{Sb}$. La Figura 3 muestra las señales de ATD para los dos compuestos empleados. En la Figura 3a, que representa el termograma para para el $\ln _{0,80} \mathrm{Mn}_{0,20} \mathrm{Sb}$, se pueden observar las transiciones de fase tanto en calentamiento (fusión) como en enfriamiento (solidificación) para ambos compuestos (InSb y MnSb), ubicándose como pares de picos tanto en la región de temperatura ascendente como en la descendente. Por el contrario, en la Figura $3 \mathrm{~b}$ $\left(\mathrm{In}_{0,90} \mathrm{Mn}_{0,10} \mathrm{Sb}\right)$, se observa un solo pico en la región de calentamiento, mientras que en la de enfriamiento se observan los dos picos, pero mucho más cercanos en comparación con la Figura 3a. 

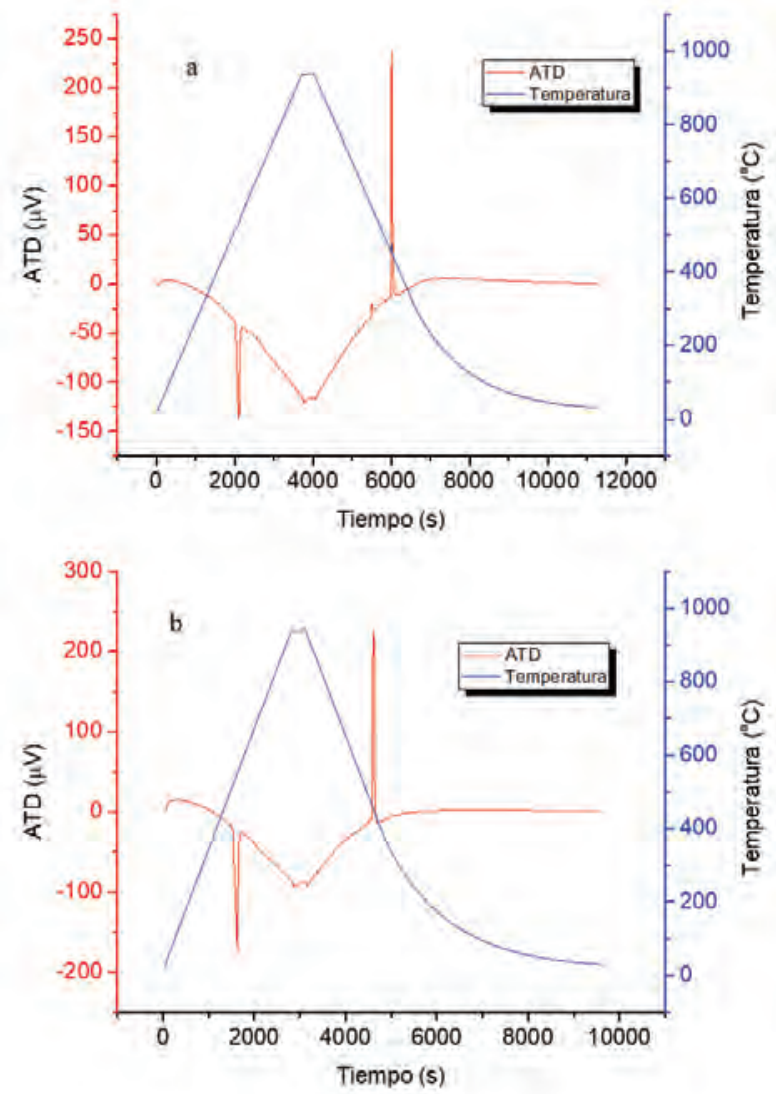

Figura 3. Señales de ATD para $\ln _{0,80} M n_{0,20} S b$ (a) y $\ln _{0,90} M n_{0,10} S b$ (b). La línea azul corresponde al valor de temperatura en la cámara y la roja a la señal diferencial

Cuando se sospecha de la presencia de picos superpuestos, como es el caso de la Figura 3 (tanto a como b), la opción experimental consiste en reducir la velocidad del barrido térmico. Esto, como se mencionó en la introducción, permite separar este tipo de señales a expensas de la cali- dad de éstas. En la Figura 3b, se muestra el ensayo con la menor tasa de calentamiento/enfriamiento posible. Se puede observar cómo, para el $\mathrm{In}_{0,90} \mathrm{Mn}_{0,10} \mathrm{Sb}$, aun cuando se estudia a muy baja velocidad de barrido es imposible lograr separar señales que sí están evidentemente separadas 
en el caso del $\ln _{0,80} \mathrm{Mn}_{0,20} \mathrm{Sb}$, aun cuando es corrido a una velocidad mayor. Es en estas situaciones donde la propuesta del presente trabajo gana valor.

Para verificar si en el pico de calentamiento del $\ln _{0,90} \mathrm{Mn}_{0,10} \mathrm{Sb}$ (Figura 3b) se está dando una transición de fase simultánea para ambos compuestos, se estudia la derivada de la señal de ATD, la cual puede ser observada en la Figura 4, donde se presenta en detalle la sección correspondiente al calentamiento de la muestra (Figura 4b), observándose la presencia de un doble pico en la derivada temporal de la señal.
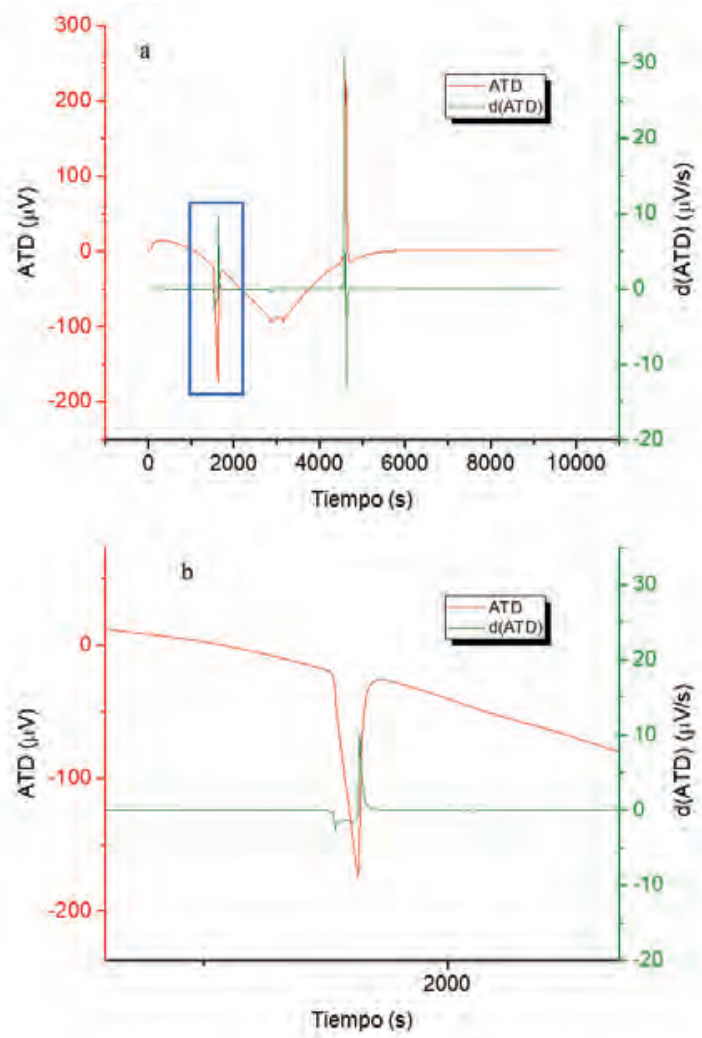

Figura 4. (a) Señal de ATD para el $\ln _{0,90} \mathrm{Mn}_{0,10} \mathrm{Sb}$.

La línea verde representa la derivada temporal de la señal de ATD (d(ATD)).

La Figura 4b muestra el detalle marcado en el rectángulo azul en (a) 
Ajustes por funciones de Gumbel

Valiéndose de que los picos correspondientes a las transiciones de fase de los dos materiales se encuentran claramente diferenciados para el $\mathrm{In}_{0,80} \mathrm{Mn}_{0,20} \mathrm{Sb}$ (Figura 3a), se emplearon funciones de Gumbel para ajustar estos picos y probar la efectividad de éstas para tal fin. La Figura 5 presenta un pico en la transición de fusión (calentamiento, Figura 5a) y uno en la transición de solidificación (enfriamiento, Figura 5b) para el ATD correspondiente al $\ln _{0,80} \mathrm{Mn}_{0,20} \mathrm{Sb}$, cada uno con su respectivo ajuste empleando una función de Gumbel.
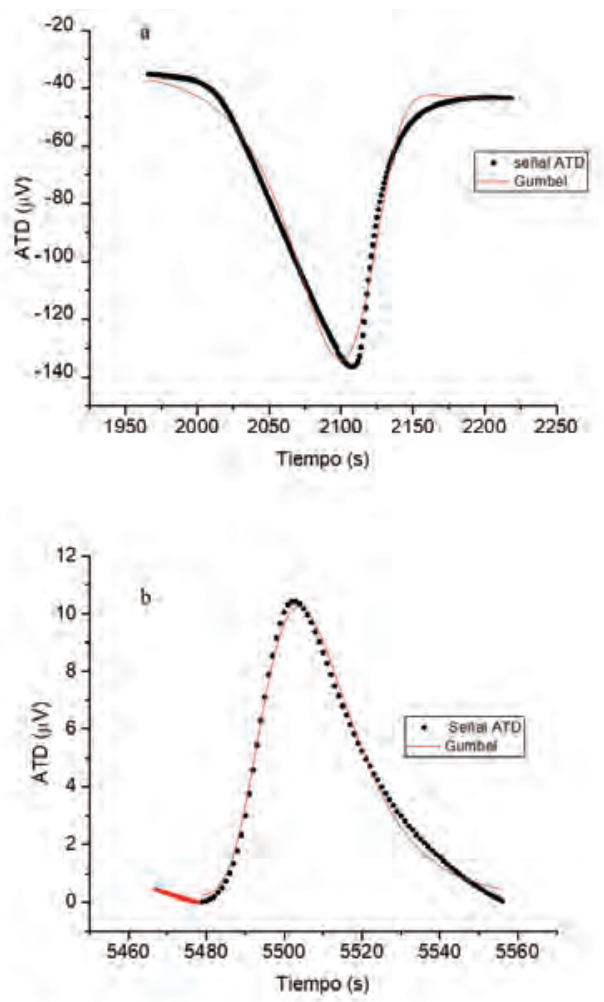

Figura 5. Detalle de los picos correspondientes a una transición de fase única de calentamiento (a), y de enfriamiento (b), para el $\ln _{0,80} M_{0,20} S b$.

Los puntos corresponden a datos experimentales, la línea roja corresponde al ajuste realizado con funciones de Gumbel, para (a), $\alpha=118 \mathrm{~s}, \beta=27,38 \mathrm{~s}^{-1}$, $R^{2}=0,984$, y para (b), $\alpha=5504 \mathrm{~s}, \beta=11,24 \mathrm{~s}^{-1}, \mathrm{R}^{2}=0,993$ 
Como ya se ha mencionado, las mediciones de ATD no son solo útiles para determinar procesos térmicos en un material, sino que, a partir del área bajo la curva de estos picos, y disponiendo de estándares de alta pureza para la calibración del equipo, es posible determinar la entalpía de fusión (solidificación) de los materiales en estudio (Borchardt \& Daniels, 1957). Esta información es de particular interés si se desea, por ejemplo, estimar costos de producción en procesos industriales. Al conocer la energía necesaria para inducir la transformación deseada de cada gramo de material, se puede tener idea del costo en energía (y por tanto en dinero) que conllevará su producción en masa. En este punto gana importancia la utilización de las funciones de Gumbel, ya que partiendo de que las señales correspondientes a los cambios de fase (superpuestas) siguen este comportamiento, se podrán identificar los dos picos superpuestos y posteriormente determinar las respectivas áreas. La Figura 6 muestra el resultado de aplicar las funciones de Gumbel para ajustar el pico obtenido experimentalmente en la transición de calentamiento para el $\mathrm{In}_{0,90} \mathrm{Mn}_{0,10} \mathrm{Sb}$, el cual contiene de forma superpuesta la contribución de las señales del InSb y el MnSb, permitiendo obtener información acerca de las dos transiciones de fase por separado.

Para poder determinar entalpías de fusión de los compuestos $(\Delta H)$, se emplea el modelo de Borchardt-Daniels (Borchardt \& Daniels, 1957), en el cual, si se considera que no hay cambios en la capacidad calorífica de los materiales durante el cambio de fase, la entalpía de fusión de un material será proporcional al área bajo la curva del pico correspondiente durante el proceso de transformación, esto se resume en la ecuación (2):

$$
\Delta H=-K \int_{t_{0}}^{t_{f}} \Delta T d t=-K A
$$

donde $A$ será el área bajo la curva y $K$ una constante de calibración para el equipo de ATD utilizado, que depende del valor de temperatura máxima del pico. 


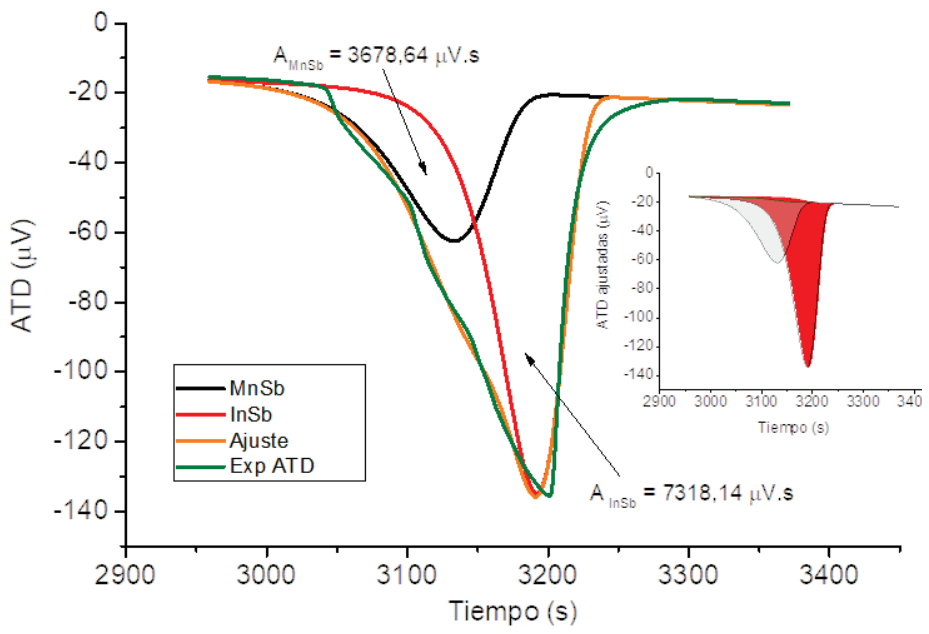

Figura 6 Detalle del pico de calentamiento para el $\ln _{0,90} \mathrm{Mn}_{0,10} \mathrm{Sb}$, donde ya se ha detectado las transiciones de fase simultáneas. La línea verde corresponde a los datos experimentales de ATD, las líneas roja y negra corresponden a las dos señales separadas InSb y MnSb respectivamente y, la naranja a la suma de estos dos ajustes $\left(R^{2}=0,992\right)$. En el inserto se detallan las áreas consideradas para cada señal aislada

Partir de que el material no cambia su capacidad calórica durante el cambio de fase es un supuesto no necesariamente cierto, para subsanar esto se implementó el método propuesto por Cavalguera y colaboradores (Clavaguera, Clavaguera-Mora, \& Fontana, 1998) en el que, a partir de un método iterativo, plantea la substracción a la señal de una línea base que depende de la fracción de material transformado (y que ha cambiado su capacidad calórica).
A partir de la información de temperatura en el ATD y la deconvolución lograda por medio de los ajustes, se pueden determinar las temperaturas a las cuales se obtienen los picos para el MnSb y el InSb, obteniéndose respectivamente: $\mathrm{T}_{\mathrm{MnSb}}=509{ }^{\circ} \mathrm{C} \mathrm{y}$ $\mathrm{T}$ InSb $=528{ }^{\circ} \mathrm{C}$. Finalmente siguiendo el método antes propuesto para compensar el cambio en la capacidad calorífica (Clavaguera et al., 1998), se determinaron las líneas base y se calcularon las áreas bajo las curvas utilizando métodos discretos implemen- 
tados en Python. Las áreas determinadas, con sus respectivas líneas base pueden ser observadas en el inserto de la Figura 6, donde se obtuvo para el InSb. AlnSb $=7318,14 \mu \mathrm{V} \cdot \mathrm{s}$ y para el de $\mathrm{MnSb}, \mathrm{A}_{\mathrm{MnSb}}=3678,64 \mu \mathrm{V} \cdot \mathrm{s}$.

\section{DISCUSIÓN}

\section{Análisis de rayos $X$}

En la Figura 2 se muestran los índices de Miller de las reflexiones indexadas, correspondientes al InSb dopado con Mn. Se obtuvo una figura de mérito $M(10)=680$ (Razeghi, 2004; Verma et al., 2011). Las reflexiones no indexadas pueden ser atribuidas al MnSb rico en antimonio (Novotortsev et al., 2011).

\section{Señales de ATD simultáneas}

El doble pico para la derivada de la señal de ATD (Figura 4b) evidencia dos tasas cambio diferentes en la velocidad de calentamiento de la muestra, lo que indica la presencia de dos transiciones de fase, donde una de mayor intensidad (asociada a la fusión del InSb) apantalla la señal de la segunda (asociada a la fusión de los agregados de InMn).

\section{Ajustes por funciones de Gumbel}

Para el pico de calentamiento presentado en la Figura 5a, la curva de datos es ajustada con un coeficiente de correlación $\mathrm{R}^{2}=0,984$, mientras que para la curva de enfriamiento presentada en la Figura 5b el ajuste arrojó un coeficiente de correlación $\mathrm{R}^{2}=0,993$. Ambos valores del coeficiente de correlación indican que las funciones de Gumbel son bastante adecuadas para modelar la forma de los picos de ATD, esto también se evidencia en los dos gráficos de la Figura 5, donde las líneas de ajuste se ciñen a los datos experimentales.

En la Figura 6 se pueden evidenciar dos picos de tamaño muy diferente (altura y área), lo que sugiere la presencia de un material en mayor proporción que otro, resultado que era de esperar a partir de la información obtenida de la Figura 3, donde los picos estaban claramente separados, o si simplemente se considera la pro- 
porción mayor en que se encuentra el InSb frente al MnSb. Todo esto sugiere que los picos deconvolucionados, a partir de las funciones de Gumbel sí son representativos de lo que ocurre en el material durante el proceso de trasformación (fusión en este caso).

No es intención de este trabajo explorar los valores para las entalpías de los compuestos en estudio, ya que estos son utilizados únicamente como modelos para demostrar las bondades del uso de las funciones de
Gumbel en el análisis de picos de ATD, adicionalmente, analizar este tipo de resultados implicaría un conocimiento más profundo de las características químicas y estructurales de cada compuesto y, de cómo sus diferentes fases interactúan entre sí, lo que requeriría del análisis por otras técnicas que provean información adicional, como microscopía electrónica con técnicas como barridos convencionales o electrones retrodispersados, lo cual se desvía del objetivo principal de este trabajo.

\section{CONCLUSIÓN}

Se ha encontrado que la función de Gumbel ajusta correctamente la forma con gran asimetría de las curvas de ATD obtenidas para las aleaciones estudiadas. Esto permite la separación de procesos simultáneos y el cálculo de valores asociados a cada proceso. Los coeficientes de correlación lineal $\left(\mathrm{R}^{2}\right)$ obtenidos son superiores a 0,98 , estos altos valores obtenidos para $\mathrm{R}^{2}$ indican que las funciones representan muy bien matemáticamente el contorno de los picos de ATD trabajados.
Al disponer de una forma matemática adecuada para representar los picos de ATD, se pueden emplear estas funciones para deconvolucionar señales de ATD que sean producto de múltiples transiciones de fase, que ocurran en intervalos de temperatura simultáneos y que normalmente son enmascaradas unas con otras. Esto permite obtener información, como las áreas de los picos o la temperatura de cambio de fase de cada compuesto, información que actualmente es de muy difícil acceso en el caso de compuestos mezclados con transiciones de fase simultáneas. 


\section{LISTA DE REFERENCIAS}

Akash, M. S. H., \& Rehman, K. (2020). Differential Thermal Analysis Essentials of Pharmaceutical Analysis (pp. 207-213): Springer.

Awschalom, D. D., Loss, D., \& Samarth, N. (2013). Semiconductor spintronics and quantum computation: Springer Science \& Business Media.

Borchardt, H. J., \& Daniels, F. (1957). The application of differential thermal analysis to the study of reaction kinetics1. Journal of the American Chemical Society, 79(1), 41-46. doi: 10.1021/ja01558a009

Clavaguera, N., Clavaguera-Mora, M., \& Fontana, M. (1998). Accuracy in the experimental calorimetric study of the crystallization kinetics and predictive transformation diagrams:

Application to a Ga-Te amorphous alloy. Journal of Materials research, 13(3), 744-753. doi: 10.1557/JMR.1998.0094

Chang, K.-H. (2015). Chapter 10 - Reliability Analysis. In K.-H. Chang (Ed.), e-Design (pp. 523-595). Boston: Academic Press.

Charsley, E., Price, D., Hunter, N., Gabbott, P., Kett, V., Gaisford, S., . . . Scowen, I. (2019). Principles of thermal analysis and calorimetry: Royal society of chemistry.

Gorgoso-Varela, J. J., \& Rojo-Alboreca, A. (2014). Use of Gumbel and Weibull functions to model extreme values of diameter distributions in forest stands. Annals of forest science, 71(7), 741-750. doi: 10.1007/s13595-014-0369-1

Gumbel, E. J. (1954). Statistical theory of extreme values and some practical applications. NBS Applied Mathematics Series, 33.

Hershfield, D. M. (1973). On the probability of extreme rainfall events. Bulletin of the American Meteorological Society, 54(10), 1013-1018. doi: 10.1016/j.ejrh.2015 .06.007 
Janssen, Y., Nicasio, J., Dudley, K., Xia, B., \& Simonson, J. (2020). Development of insitu differential thermal analysis for crystal growth experiments. Bulletin of the American Physical Society.

Katayama-Yoshida, H., \& Sato, K. (2003). Spin and charge control method of ternary II$\mathrm{VI}$ and III-V magnetic semiconductors for spintronics: theory vs. experiment. Journal of Physics and Chemistry of Solids, 64(9-10), 1447-1452. doi: 10.1016/S0022-3697(03)00126-4

Kramer, E. (1992). Materials Science and Technology: A Comprehensive Treatment (Vol. 5: Phase Transformations in Materials).

Liu, C., Li, Y., \& Zeng, Y. (2010). Progress in antimonide based III-V compound semiconductors and devices. Engineering, 2(08), 617. doi: 10.4236/eng.2010.28079

Nadarajah, S. (2006). The exponentiated Gumbel distribution with climate application. Environmetrics: The official journal of the International Environmetrics Society, 17(1), 13-23. doi: 10.1002/env.739

Novotortsev, V., Kochura, A., Marenkin, S., Fedorchenko, I., Drogunov, S., Lashkul, A., \& Lähderanta, E. (2011). Synthesis and magnetic properties of the InSb-MnSb eutectic. Russian Journal of Inorganic Chemistry, 56(12), 1951-1956. doi: $10.1134 /$ S0036023611120400

Razeghi, M. (2004). Antimony Based Materials for Electro-Optics Semiconductor Nanostructures for Optoelectronic Applications.

Roisnel, T., \& Rodríquez-Carvajal, J. (2001). WinPLOTR: a windows tool for powder diffraction pattern analysis. Paper presented at the Materials Science Forum.

Školáková, A., Pr־ša, F., \& Novák, P. (2020). Thermal analysis of FeAl intermetallic compound sintered at heating rate of $300^{\circ} \mathrm{C} / \mathrm{min}$. Journal of Alloys and Compounds, 819, 152978. doi: 10.1016/j.jallcom.2019.152978

Verma, U., Devi, N., Sharma, S., \& Jensen, P. (2011). Spin-polarized first-principles study of ferromagnetism in zinc-blende In $1-\mathrm{x} \mathrm{Mn} \times \mathrm{Sb}$. The European Physical Journal B, 81(4), 381-386. doi: 10.1140/epjb/e2011-20047-8 